\title{
Simultaneous processing of verbal and spatial information
}

\author{
TIMOTHY A. SALTHOUSE \\ University of Michigan, Ann Arbor, Michigan 48104
}

\begin{abstract}
Two experiments investigated the generality of the selective interference phenomenon by studying several different concurrent tasks, each involving either verbal or spatial information, and a range of retention intervals from 10 to $30 \mathrm{sec}$. The results supported the selective interference predictions, since the interference in performance was greater when the two simultaneous tasks both involved either verbal information or spatial information than when one of the tasks involved verbal and the other spatial information. It was concluded that not only was the selective interference phenomenon a rather general effect but that, since it was unaffected by changes in the nature of the component tasks, it was also probably caused by central processing capacity limitations rather than by competition for a specific structural mechanism.
\end{abstract}

In a recent study by the author (Salthouse, 1974), it was found that two memory tasks could be handled simultaneously with little or no performance interference if the two tasks involved different types of information. Recognition memory tasks with nonverbal or verbal stimuli were performed during the retention interval of a recall memory task consisting of either spatial or verbal information. The interference in performance when the two memory tasks were performed concurrently was highly selective in nature, with the most interference resulting when the two tasks both involved either verbal information or spatial information. Primarily because of this pattern of selective interference, it was suggested that spatial and verbal information are processed and stored in separate processing systems.

Since the separate processing system hypothesis is potentially of great theoretical importance, the current experiments were designed to investigate more thoroughly the simultaneous processing of spatial and verbal information. Tasks with both lesser and greater memory requirements than the recognition memory tasks in the earlier experiments and covering a range of retention intervals from 10 to $30 \mathrm{sec}$ were studied in the present experiments. These manipulations were intended to assess the generality of the selective interference phenomenon and to determine whether it is limited to a particular combination of memory tasks or to a particular time span in memory.

The data obtained from the memory demand and retention interval manipulations should also be useful in making inferences about the reasons for the selective interference phenomenon. The nature of the interference effect should be inferrable if two tasks with different emphases are investigated in the same experimental situation. If the interference is similar in direction and magnitude with both tasks, one can conclude that the interference is caused by a nonspecific limitation in overall processing capacity. On the other hand, if the interference is only evident with one of the tasks, then one can conclude that the interference is structural, i.e., the result of competition for a specific processing mechanism.

Both of the current experiments utilize the same stimuli, consisting of arrays of 25 items with seven circled target items, that were used in the earlier study (Salthouse, 1974). The stimuli have the unique advantage of possessing the capability for differing in the type of information the subject is required to remember, while remaining the same in the means of presentation and in the formal structure of the stimuli. The differing types of information are produced by instructing the subject to remember the identities of the target items for verbal information and to remember the positions of the target items for spatial information.

\section{EXPERIMENT I}

In the first experiment, verbal and spatial activities selected to require very little memory involvement were performed during the interval in which the subject was remembering verbal or spatial information from the array stimuli. The activities were counting backward by threes and a classification task requiring "mental rotation." Three retention intervals were also studied: 10,20 , and $30 \mathrm{sec}$.

The expectations from the hypothesis that selective interference is a general, rather than a mechanism-specific, effect were: (a) that the type of retention interval activity should interact with the type of recall information and (b) that this interaction would not be affected by the length of the retention interval.

\footnotetext{
Method

Subjects. Thirty-six college-age subjects received $\$ 2$ for their participation in an experimental session lasting approximately $1 \mathrm{~h}$. The subjects were alternately assigned to one of two groups in the order they reported for testing. The final composition of each group consisted of 6 males and 12 females.

Materials. The stimuli from which the subject was to remember the verbal or spatial information are described and
} 
illustrated in Salthouse (1974). Briefly, they were yellow cards upon which were typed diamond-shaped arrays of the 25 numbers from 1 to 25 , with seven numbers circled in red ink. The subject was required to remember either the identities or the positions of the seven circled target items. Recall consisted of the reproduction of the target items for the identity information and circling the dots in a diamond-shaped array of dots whose positions corresponded to the positions of the target items for the position information. For each subject a different array was presented on every trial.

The material for the spatial interpolated task consisted of pages containing 72 pairs of characters in various orientations. One half of the pairs had the same character as both members of the pair. The other half had an alphabetic character (F, G, or R) as one member of the pair and its mirror image as the other. The important manipulation that insured that this task involved spatial information was the relationship between the orientations of the two members in each pair. The members differed in their relative orientations by 60,120 , or $180 \mathrm{deg}$, depending upon the page. Thus, in order to classify a character pair as same (i.e., identical characters in different orientations), the subject had to "mentally rotate" one of the characters to match the orientation of the other character in the pair. Evidence from several studies (e.g., Cooper \& Shepard, 1973; Shepard \& Metzler, 1971) strongly suggests that this mental rotation process involves the transformation of imaginal or spatial information.

Procedure. Two groups of subjects were each presented with nine conditions composed of the factorial combination of three retention intervals and three types of interpolated activity. The two groups were distinguished on the basis of whether subjects were instructed to remember verbal information (Group V) or spatial information (Group S). The three retention intervals were 10,20 , and $30 \mathrm{sec}$, and the three types of interpolated activity were none, auditory-verbal, and visual-spatial.

The auditory-verbal interpolated activity was based on a task originally introduced by Peterson and Peterson (1959). Immediately after the presentation of the stimulus array, the subject was read a three-digit number from which he was to count backward by threes until he received a signal to stop counting and recall the remembered information.

The visual-spatial interpolated activity consisted of written same/different classification judgments about pairs of printed characters displayed in different orientations. Prior to each trial, the subject was given a new page of character pairs and instructed to begin classifying the pairs by writing an $S$ or a $D$ adjacent to each pair immediately after the presentation of the stimulus array and to continue classifying until the signal for recall.

Performance on the counting backward task was not monitored. Classification performance was measured on the mental rotation task, but no differences were apparent across conditions or groups and, hence, the results are not reported.

Six trials were presented in each of the nine conditions to each subject. Trials were blocked according to the type of interpolated activity, and within each block the three retention intervals were distributed equally. The order of the three blocks of trials was counterbalanced across subjects in each group. Between successive blocks, subjects were given a rest period of approximately $3 \mathrm{~min}$.

On a given trial in the experiment, the subject first inspected the array of 25 numbers for $4 \mathrm{sec}$, attempting to remember either the identities (Group V) or the positions (Group S) of the seven target items. Next, the subject either performed the mental rotation task, performed the counting backward task, or merely rested for an interval of 10,20 , or $30 \mathrm{sec}$. At the sound of a buzzer signaling the end of the retention interval, the subject began recalling the information from the stimulus array, eventually producing seven responses even if some were mere guesses. (a)

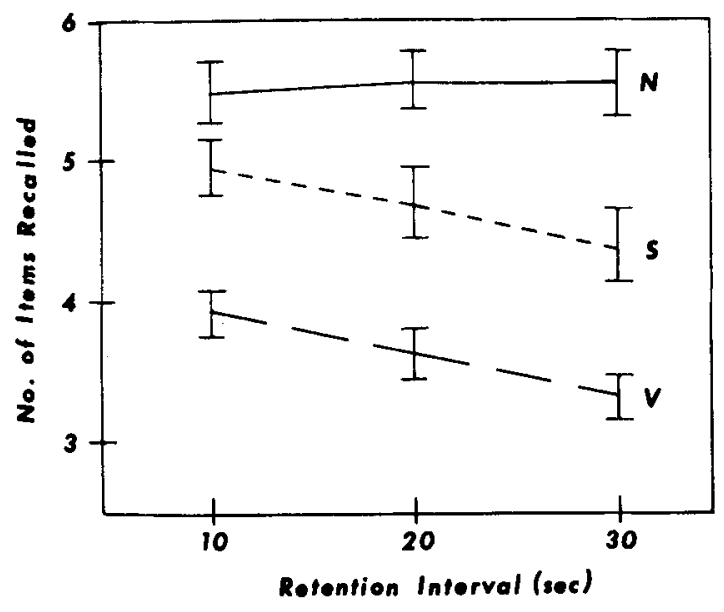

(b)

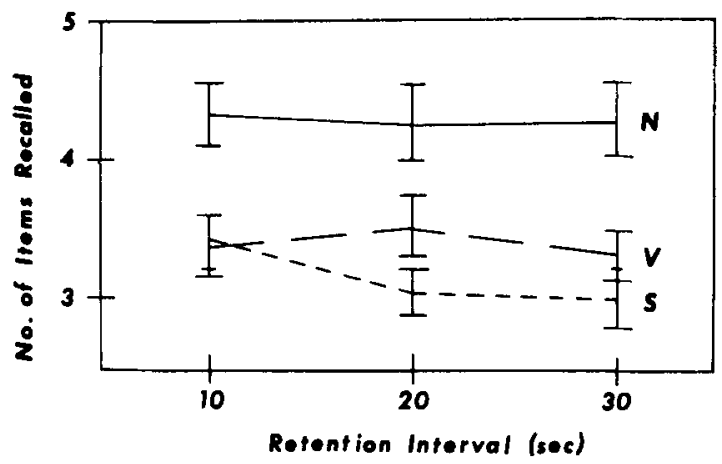

Figure 1. (a) Verbal recall performance as a function of retention interval and type of interpolated activity. (b) Spatial recall performance as a function of retention interval and ty pe of interpolated activity. $N, V$, and $S$ denote none, verbal, and spatial interpolated activities. The bars above and below each point represent the standard errors.

\section{Results}

The dependent variable of interest is the number of verbal or spatial items correctly recalled. The means and standard errors of this measure for all conditions are illustrated in Figures $1 \mathrm{a}$ and $1 \mathrm{~b}$. Figure $1 \mathrm{a}$ contains the data from Group $\mathrm{V}$, and Figure $1 \mathrm{~b}$ contains the data from Group $S$.

The initial impressions conveyed by Figures $1 \mathrm{a}$ and $1 \mathrm{~b}$ are supported statistically by the outcome of an analysis of variance. Target identities are remembered much better than target positions $[\mathrm{F}(1,34)=2.41, \mathrm{p}<.001]$, short retention intervals lead to better recall than long retention intervals $[\mathrm{F}(2,68)=5.46, \mathrm{p}<.01]$, and memory intervals with no interpolated activity result in a greater number of items recalled than memory intervals in which an interpolated task was performed $[\mathrm{F}(2,68)=58.95, \mathrm{p}<.001]$. Furthermore, the interaction of Type of Recall Information by Type of Interpolated Activity was significant $[F(2,68)=13.45$, 
$p<.0011$, but none of the other interactions, including the three-way interaction among type of recall information, type of interpolated activity, and retention interval, was statistically significant.

The most important of the results are the findings that the auditory-verbal interpolated task causes more loss of verbal information than of spatial information, while the reverse is true for the visual-spatial interpolated task, and that this selective interference relationship is maintained, with one exception, throughout all retention intervals.

The trends apparent in the group data were also consistent across subjects. All 18 of the subjects remembering verbal information recalled more items when performing the mental rotation interpolated activity. However, the pattern was reversed when spatial information was remembered, as 13 of the 18 subjects exhibited better recall with the counting backward task than with the mental rotation task.

At the conclusion of the experiment, each subject ranked the difficulty of the three interpolated activity conditions. One hundred percent of the Group V subjects rated the counting backward task as the most difficult, while only $61 \%$ of the Group S subjects rated it as most difficult. The difference between the percentages is statistically significant $(p<.01)$ and indicates that subjects are subjectively aware of the selective interference phenomenon, although they generally found it more difficult to perform the counting backward task than to perform the mental rotation classification task.

\section{EXPERIMENT II}

In the second experiment, verbal and spatial tasks, chosen because of their demanding memory requirements, were performed during the interval in which the subject was remembering verbal or spatial information from the array stimuli. These tasks were verbal and spatial recall tasks very similar in nature to the original recall tasks.

The major prediction from the limited-capacity interpretation of the selective interference phenomenon was that a significant interaction would result between type of recail information from the first array and type of recall information from the second array. Also of interest was the relationship between the performances on the two concurrent recall tasks.

\section{Method \\ Subjects. Thirty-two college-age subjects received $\$ 3$ for their participation in a session lasting approximately $1.5 \mathrm{~h}$. The subjects were alternately assigned to one of two groups in the order they reported for teating. The final compostion of each group was 9 females and 7 males for Group $\$$ and 11 females and 5 males for Group V.}

Materinla. Two types of 25-item arrays were used as stimuli. One type was the yellow cards with the diumond-shaped atrays of numbers described in the previous experiment. The second type consisted of square-shaped arrays of uppercase alphabetic characters typed on white cards. All of the letters of the alphabet except " $O$ " were used in the letter arrays, and they were randomly located throughout the array with changed positions on each array. As with the number arrays, seven of the items were circled in red ink and served as the target items. The mode of response for both types of stimuli was similar to that described in the previous experiment, with the exception that the position responses for the square-shaped arrays were made on a square-shaped rather than a diamond-shaped array of dots.

Procedure. Two groups of subjects were distinguished on the basis of the type of information they were required to remember from the square-shaped array of letters. The group remembering the target identities, i.e., verbal information, was designated Group V, and the group remembering the target positions, i.e., spatial information, was designated Group $S$.

In each group, five experimental conditions were produced by combinations of two separate recall tasks. One of the recall tasks involved remembering information from the square-shaped letter arrays for a duration of 20 sec. The other recall task involved remembering information from the diamond-shaped number arrays for a duration of $5 \mathrm{sec}$. Within the two groups, the five conditions were identical except that the Group V subjects remembered verbal information (i.e., target identities) from the square-shaped letter arrays, whereas the Group S subjects remembered spatial information (i.e., target positions).

The labeling of the conditions was derived by designating the type of information remembered from the square-shaped letter arrays with an uppercase letter (i.e., either $V$ or $S$ for verbal or spatial information) and the type of information remembered from the diamond-shaped number arrays with a lowercase letter (i.e., either $v$ or $s$ for verbal or spatial information). The five conditions in Group V were thus called V when subjects remembered verbal information from the square letter arrays for $20 \mathrm{sec}, \mathrm{v}$ when subjects remembered verbal information from the diamond number arrays for $5 \mathrm{sec}, \mathrm{s}$ when subjects remembered spatial information from the diamond number arrays for $5 \mathrm{sec}$, $V_{v}$ when subjects remembered verbal information from the diamond number arrays for $5 \mathrm{sec}$ while simultaneously remembering verbal information from the square letter arrays for a duration of $20 \mathrm{sec}$, and Vs when subjects remembered spatial information from the diamond number arrays for $5 \mathrm{sec}$ while simultaneously remembering verbal information from the square letter arrays for a duration of $20 \mathrm{sec}$. The five conditions in Group S were identical, except that the subjects remembered spatial information from the square-shaped letter arrays and, hence, Conditions $\mathrm{V}, \mathrm{Vv}$, and $\mathrm{V}_{\mathrm{s}}$ were labeled $\mathrm{S}, \mathrm{Sv}$, and $\mathrm{Ss}$, respectively.

An outline of the recall tasks involved in the five conditions in each group is provided in Table 1 . With the aid of the outline, it can be seen that in each group Conditions Vv, Vs, Sv, and Ss are the experimental conditions of interest (i.e., they involve two concurrent recall tasks), while the other conditions are control conditions designed to provide estimates of recall performance when only one task was required on each trial.

In an attempt to equate the attention demands and the degree of response involvement across Conditions $\mathrm{V}, \mathrm{Vv}$, and $\mathrm{Vs}$ and $\mathrm{S}$, $\mathrm{Sv}$, and $\mathrm{Ss}$, a distractor task was introduced into the retention interval of Conditions $V$ and $S$. The task required subjects to inspect a diamond-shaped number array stimulus card, to count the number of corner items that were circled in that array, and then to write that number on the response form. This task was designed to involve some of the same aspects of attending to the intervening array and responding to that information that were involved in the recall tasks found in Conditions $\mathrm{Vv}, \mathrm{V}_{\mathrm{s}}, \mathrm{Sv}$, and Ss, but without requiring any substantial memory component

All subjects were instructed to guess, if necessary, to produce seven responses on each trial. Two special instructions preceded 
the dual-task conditions (i.e., Vv, Vs, Sv, and Ss). The first emphasized the necessity of responding rapidly to the second recall task. This instruction was apparently successful, as in nearly all of the trials the recall of information from the diamond number arrays had been completed before the arrival of the signal indicating that the square-shaped letter-array information was to be recalled. A second instruction informed subjects that, if they began to have difficulty handling both tasks at once, they should emphasize the recall task with the square letter arrays rather than the recall task with the diamond number arrays. The purpose of this instruction was to try to keep the motivational emphasis on the square-shaped letter-array recall task constant across Conditions V, Vv, and Vs and S, Sv, and Ss.

All 20 trials within a condition were blocked together, and instructions preceding each block clearly stated what type of information the subject was to remember from each stimulus. The order in which the subjects in each group received the five conditions was counterbalanced such that across subjects each condition was presented in the same average order.

The actual structure of a trial in one of the more complex conditions, Vs, was as follows. First, the subject inspected the square letter array on a white card for approximately $4 \mathrm{sec}$, during which time he attempted to remember the identities of the circled target letters. Several seconds later, the onset of a clicking sound signaled the subject to begin inspecting the diamond number array on a yellow card, which he did throughout the 4-sec duration of the clicking sound. Since this was Condition Vs, the subject was attempting to remember the positions of the circled numbers from the diamond number array. Five seconds after the subject had 'stopped inspecting the diamond number array, a buzzer sounded signaling the subject to begin recalling the position information from that array. (Note that the last information presented on a trial was the first to be recalled!) Finally, $20 \mathrm{sec}$ after the subject had finished inspecting the square letter array, he received a signal to begin recalling the identity information from that array. In the other conditions the time durations remained the same but the number of tasks involved and the type of information remembered from each array varied.

\section{Results}

As in the previous experiment, the major dependent variable of interest was the mean number of verbal and spatial items correctly recalled in each condition. Recall performance for the two groups in the square letter-array task and in the diamond number-array task are presented in Table 1.

The major comparisons in Table 1 are among the recall performances in Conditions $\mathrm{Vv}, \mathrm{Vs}, \mathrm{Sv}$, and $\mathrm{Ss}$. Separate analyses of variance were conducted on the data in these conditions for both the square-shaped letter-array recall task and the diamond-shaped number-array recall task. The primary result of interest from these analyses was that the interaction between type of information in the square-shaped letter-array task and type of information in the diamond-shaped number-array task was significant in both the square-shaped letter-array data $[F(1,30)=16.15$, $\mathrm{p}<.001]$ and the diamond-shaped number-array data $[F(1,30)=5.89, p<.05]$. Identical statistical results were obtained when the recall scores in Conditions Vv, Vs, Sv, and Ss were "conditionalized" with respect to the scores in the appropriate control conditions for each group. That is, the outcomes from the analyses of variance were the same when the data were ratios in the form $(\mathrm{Vv}-\mathrm{V}) / \mathrm{V}$ or $(\mathrm{Ss}-\mathrm{s}) / \mathrm{s}$ as when the data were simply the absolute number of items recalled in the experimental conditions. Inspection of the data in the rightmost two columns of Table 1 indicates the direction of the interaction: Performance on either recall task is clearly better if the other information being remembered concurrently involves a different type of information.

The finding that the interactions were significant in the performance of both recall tasks greatly increases the reliability of the effect, since the two tasks were distinct and at least conceptually independent. [Indeed, if one were to assume that performance on the two tasks was completely independent, one could estimate the probability that the combined interaction effect resulted from chance as being the product of the individual probabilities, i.e., $(p<.001) \times(p<.05)=p<.00005$ !]

In order to determine the extent to which recall performance on one of the two tasks was related to recall performance on the other stimulus task, correlation coefficients were computed between the levels of performance on the two concurrent tasks. Two methods were used: (a) calculating a single correlation coefficient for each condition using the mean score for each subject and (b) calculating a separate correlation coefficient for each subject by correlating that subject's scores on the two recall tasks for each trial in the condition. For none of the conditions were the correlations computed by Method a or the mean correlations computed by Method b significantly negative, as one might expect from a limited-capacity processing system notion. Similar results were obtained in the earlier experiments by the author (Salthouse, 1974), but an explanation is not readily apparent.

Correlations between mean recall performances in Conditions $\mathrm{v}$ and $\mathrm{s}, \mathrm{V}$ and $\mathrm{v}$, and $\mathrm{S}$ and $\mathrm{s}$ were: $\mathrm{v}-\mathrm{s}$, $\mathrm{r}=+.493(\mathrm{p}<.01) ; \mathrm{V} \cdot \mathrm{v}, \mathrm{r}=+.695(\mathrm{p}<.01)$; and $\mathrm{S}-\mathrm{s}$, $\mathrm{r}=+.462$ (n.s.).

Table 1

Verbal and Spatial Recall Performance in the Two Recall Tasks in Experiment II

\begin{tabular}{llllll}
\hline & \multicolumn{5}{c}{ Condition } \\
\cline { 2 - 5 } Recall Task & $\mathrm{V}$ & $\mathrm{v}$ & $\mathrm{s}$ & $\mathrm{Vv}$ & $\mathrm{Vs}$ \\
\hline
\end{tabular}

Group V

\begin{tabular}{llllll}
$\begin{array}{l}\text { Square-shaped } \\
\text { letter arrays }\end{array}$ & 5.66 & & & 4.43 & 5.11 \\
Diamond-shaped & $(.20)$ & & & $(.20)$ & $(.25)$ \\
number arrays & & 5.43 & 4.17 & 4.99 & 3.85 \\
& & $(.17)$ & $(.10)$ & $(.22)$ & $(.11)$ \\
\hline & $\mathrm{S}$ & $\mathrm{v}$ & $\mathrm{s}$ & $\mathrm{Sv}$ & $\mathrm{Ss}$ \\
\hline
\end{tabular}

Group S

$\begin{array}{llllll}\text { Square-shaped } & 3.87 & & & 3.38 & 3.18 \\ \text { letter arrays } & (.25) & & & (.19) & (.22) \\ \text { Diamond-shaped } & & 5.20 & 4.25 & 5.20 & 3.69\end{array}$

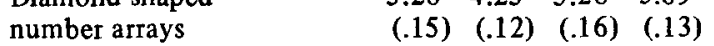

Note-Numbers in parentheses are the standard errors of the mean in each condition. 
In the current experiment, as in the previous one, the subjects ranked the conditions according to their difficulty at the end of the experimental session. However, the results in the present experiment, while in the expected direction, were not statistically significant.

\section{GENERAL DISCUSSION}

The major findings in the current experiments are the selective interference pattern of results (i.e., the significant interactions between type of information in the task presented first and type of interpolated task information) with both high- and low-demand memory activities and over a range of retention intervals from 10 to $30 \mathrm{sec}$. The apparent generality of the selective interference phenomenon suggests that it is not the result of competition for a single structural mechanism with a particular time constraint, but rather is caused by a general capacity limitation.

The results of experiments by other researchers also attest to the generality and reliability of the selective interference phenomenon (e.g., Brooks, 1967, 1968; den Heyer \& Barrett, 1971; Rollins \& Thibadeau, 1973). That the interference in performance is selective and maximal when the two concurrent tasks involve the same type information seems now to be an established finding.

A remaining issue concerns the explanation for the selective interference phenomenon. One possible explanation, in accord with traditional views of memory, might maintain that the differential interference with different types of information is a result of the amount of interference being proportional to the degree of similarity between the various pieces of information. A critical problem with this form of explanation is that in these experimental situations it is either very difficult or impossible to define similarity, except with reference to what the subject does with the stimulus material, in which case the definition loses most of its meaning and all of its usefulness.

An alternative explanation is that the existence of at least partially independent verbal and spatial information processing systems is responsible for the selective interference phenomenon. A possible anatomical basis for the different systems may lie in the distinction between the two cerebral hemispheres, since many investigators (e.g., Gazzaniga, 1970; Kimura, 1973; Newcombe, 1969) have reported that the right hemisphere is apparently specialized for processing spatial information, while the left is specialized for handling verbal information.

Many questions remain unanswered concerning the separate system hypothesis, but it is interesting to note that the large interactions of the present experiments and the selective interference pattern obtained in other experiments argue for a functional independence of verbal and spatial information processing regardless of the particular interpretation of the selective interference effect.

\section{REFERENCES}

Brooks, $L$. $R$. The suppression of visualization by reading. Quarterly Journal of Experimental Psychology, 1967, 19, 289-299.

Brooks, L. R. Spatial and verbal components of the act of recall. Canadian Journal of Psychology, 1968, 22, 349-368.

Cooper, L. A., \& Shepard, R. N. Chronometric studies of the rotation of mental images. In $W$. $G$. Chase (Ed.), Visual information processing. New York: Academic Press, 1973.

den Heyer, K., \& Barrett, B. Selective loss of visual and verbal information in STM by means of visual and verbal interpolated tasks. Psychonomic Science, 1971, 25, 100-102.

Gazzaniga, M. S. The bisected brain. New York: Appleton-Century-Crofts, 1970.

Kimura, D. The asymmetry of the human brain. Scientific American, 1973, 228, 70-78.

Newcombe, F. Missile wounds of the brain: A study of psychological deficits. Oxford, England: Oxford University Press, 1969.

Peterson, L. R., \& Peterson, M. J. Short-term retention of individual items. Journal of Experimental Psychology, 1959, 58, 193-198.

Rollins, H. A., \& Thibadeau, R. The effects of auditory shadowing on recognition of information received visually. Memory Cognition, 1973, 1, 164-168.

Salthouse, T. Using selective interference to investigate spatial memory representations. Memory \& Cognition, 1974, 2, 749-757.

Shepard, R. N., \& Metzler, J. Mental rotation of three-dimensional objects. Science, 1971, 171, 701-703.

(Received for publication April 26, 1974; revision received July $17,1974$. 